\title{
Topical interventions to prevent acute radiation dermatitis in head and neck cancer patients: a systematic review
}

\author{
Elaine Barros Ferreira ${ }^{1}$. Christiane Inocêncio Vasques ${ }^{1} \cdot$ Rafael Gadia $^{2}$. \\ Raymond Javan Chan ${ }^{3}$ • Eliete Neves Silva Guerra ${ }^{1}$ Luis André Mezzomo ${ }^{4}$. \\ Graziela De Luca Canto ${ }^{4}$ - Paula Elaine Diniz dos Reis ${ }^{1}$
}

Received: 10 June 2016 / Accepted: 5 December 2016/Published online: 12 December 2016

(C) Springer-Verlag Berlin Heidelberg 2016

\begin{abstract}
Purpose The purpose of the study is to evaluate the effects of pharmacological and non-pharmacological topical controls in the prevention of radiation dermatitis.

Methods Relevant clinical trials were identified through electronic searching databases CINAHL, CENTRAL, LILACS, PubMed, Scopus, and Web of Science. Handsearching and gray literature searches were also performed to find additional references. Primary outcomes of interest were the development of radiation dermatitis and the time of occurrence of radiation dermatitis.

Results Thirteen randomized clinical trials were included in this review. The trials were published in Chinese, English, or French, from 1980 to 2015. Pharmacological interventions
\end{abstract}

Electronic supplementary material The online version of this article (doi:10.1007/s00520-016-3521-7) contains supplementary material, which is available to authorized users.

Elaine Barros Ferreira

elaine.barrosf@gmail.com

Christiane Inocêncio Vasques

chvasques@unb.br

Rafael Gadia

rafaelgadia@gmail.com

Raymond Javan Chan

raymond.chan@health.qld.gov.au

Eliete Neves Silva Guerra

elieteneves@unb.br

Luis André Mezzomo

1.mezzomo@ufsc.br

Graziela De Luca Canto

delucacanto@gmail.com used in the trials were trolamine, aloe vera, allantoin, Lianbai liquid, sucralfate, Na-sucrose octasulfate, olive oil, hialuronic acid, and dexpanthenol. Non-pharmacological topical controls were usual care/institution routine, aqueous cream, mild soap, water thermal gel, placebo, and no intervention.

Conclusions There was no strong evidence that indicates differences between topical pharmacological interventions or non-pharmacological topical controls in the prevention of acute radiation dermatitis among patients with head and neck cancer undergoing radiotherapy.

Keywords Radiodermatitis $\cdot$ Head and neck cancer $\cdot$ Skin care $\cdot$ Radiotherapy

Paula Elaine Diniz dos Reis

pauladiniz@unb.br

1 Nursing Department, School of Health Sciences, University of Brasília, Darcy Ribeiro University Campus, Asa Norte, Brasilia, Federal District, Brazil

2 Hospital Sírio Libanês, Asa Sul, Brasília, DF, Brazil

3 FACN Royal Brisbane and Women's Hospital, Metro North Hospital and Health Service, Queensland University of Technology, QLD, Kelvin Grove 4059, Australia

4 Federal University of Santa Catarina, Rua Engenheiro Agronômo Andrey Cristian Ferreira, Trindade, Florianópolis, Santa Catarina, Brazil 


\section{Introduction}

Acute radiation dermatitis is a radiation that induces injury to the epithelium and underlying structures of the skin, characterized by erythema, dry or moist desquamation, and even ulceration [1, 2]. Its onset commonly occurs within 2-3 weeks following the radiotherapy commencement [2], and it is usually observed at skin dose levels of 20-40 Gy [3]. About 80$90 \%$ of all patients with head and neck cancer undergoing radiotherapy develop radiation dermatitis [3], whereas severe skin reactions occur in approximately $25 \%$ of these patients [4].

Several factors may potentially affect skin toxicity. Radiotherapy-related factors such as total dose, fractionation, radiation energy, volume of treated regions [5], treatment duration, boost application, and treatment site have been suggested [3]. Patient-related factors depend on age, comorbid conditions, skin phototype, and genetic predisposition [5]. Furthermore, the combination of radiotherapy and chemotherapy increases skin reactions, resulting in severe xerosis, inflammation, skin thinning, and necrosis of the upper dermis and epidermis [6]. Patients with head and neck cancer are commonly treated with radical radiotherapy or chemoradiotherapy [7], which increases the likelihood to have exacerbated acute skin toxicity $[5,7]$.

Radiotherapy side effects tend to have early onset. Although mostly mild, they can become severe and significantly impair quality of life [6]. These reactions may lead to dose reduction or discontinuation of therapy, which, in turn, could be detrimental to the treatment outcome [6], particularly in head and neck cancer patients [8]. There is no evidencebased standard approach for the prevention and treatment of radiation dermatitis, although several medications have been proposed such as topical agents, dressings, and radioprotectors [5].

Previous systematic reviews have evaluated the prevention of radiation dermatitis in several irradiated areas, either simultaneously or separately [9-15]. However, to the best of our knowledge, no review has specifically assessed the use of topical interventions in the prevention of acute radiation dermatitis in head and neck cancer patients. Therefore, the main goal of this systematic review was to answer the focused question "In patients undergoing radiotherapy for head and neck cancer, what is the effect of pharmacological topical interventions compared to non-pharmacological topical controls in the prevention of acute radiation dermatitis?"

\section{Material and methods}

The reporting of this systematic review adheres to the Preferred Reporting Items for Systematic Reviews and Meta-Analyses (PRISMA) Checklist [16].

\section{Protocol and registration}

The systematic review protocol was registered at the International Prospective Register of Systematic Reviews (PROSPERO) [17], registration number CRD42015020823.

\section{Terminology definition}

Prevention of acute radiation dermatitis was defined as to prevent a reaction from occurring (yes or no) [18] and prevention of grades 1 (erythema and dry desquamation) and 2 (bright erythema and moist desquamation) according to the Radiation Therapy Oncology Group (RTOG) criteria of acute radiation dermatitis or to the Common Terminology Criteria for Adverse Events (CTCAE) criteria for dermatitis radiation $[19,20]$. Pharmacological topical interventions were considered as products that contain both the active ingredient and the vehicle, whereas non-pharmacological topical controls were considered as those that contain only the vehicle (or base) or it is placebo/usual care/no medication [21].

\section{Eligibility criteria}

Only original prospective studies in which the objective was to investigate the effects of the use of pharmacological topical interventions (compared to non-pharmacological topical controls) in the prevention of acute radiation dermatitis in patients with head and neck cancer undergoing external beam radiotherapy were included. Studies that compared topical interventions and used prevention of acute radiation dermatitis as an outcome were eligible. There were no restrictions to the year of publication or language of the study. Age of the participants, gender, previous or concurrent therapies, health status, or dosage of treatment were also not restricted.

Studies were excluded for the following reasons: (1) cobalt therapy; (2) studies that compared exclusively non-topical interventions; (3) therapeutic and not preventive interventions; (4) studies that compared two or more products containing active ingredient; (5) insufficient data on the effect of the intervention; and (6) reviews, letters, conference abstracts, personal opinions, book chapter, retrospective study, descriptive study, case reports, or cases series.

\section{Information sources and search strategy}

Studies were identified using a search strategy adapted for each electronic database, with the aid of a health sciences librarian: CINAHL EBSCO, Cochrane Central Register of Controlled Trials (CENTRAL), LILACS, PubMed, SCOPUS, and Web of Science (Appendix 1). The hand search was performed on the reference lists from the selected articles for any additional references that might have been missed in the electronic search. In addition, a gray literature search was 
performed using Google Scholar and ProQuest Dissertations and Theses databases.

After obtaining all references, duplicates were excluded by using appropriate software (EndNoteBasic $\AA$, Thomson Reuters, USA). All the electronic database searches were conducted on March 27, 2016.

\section{Study selection}

Study selection was conducted in two phases. In phase 1, two investigators (E.B.F. and P.E.D.R.) independently screened the titles and abstracts of potentially relevant studies and selected articles that appeared to meet the inclusion criteria based on their abstracts. In phase 2 , the same reviewers independently read the full text of all selected articles and excluded studies that did not meet the inclusion criteria. Any disagreements, either in the first or second phases, were resolved by discussion and mutual agreement between the two reviewers. In case a consensus could not be reached, a third author (C.I.V.) was involved to make a final decision. Studies that were excluded after full-text assessment and the reasons for their exclusion are listed in Appendix 2.

\section{Data collection process and items}

Two investigators (E.B.F. and P.E.D.R.) independently collected the data from the selected articles: study characteristics (author(s), year of publication, setting, objectives, methods), population characteristics (sample size, age), intervention characteristics (groups, follow-up period, primary outcomes, radiation dermatitis criteria, and statistical analysis), and outcome characteristics (results and main conclusion). The third author (C.I.V.) cross-checked all the retrieved information to make a final decision. If the required data were not complete, attempts were made to contact the authors to retrieve any pertinent missing information.

\section{Risk of bias in individual studies}

To assess the risk of bias of the included randomized controlled trials (RCT), it was applied the Cochrane Collaboration Risk of Bias Tool [22], including judgments about the sequence generation, allocation concealment, blinding of participants, personnel and outcome assessors, incomplete outcome data, selective reporting, and other sources of bias. The risk of bias was assessed as low, high, or unclear. Two investigators performed this process independently (E.B.F. and P.E.D.R.). Disagreements between the two reviewers were resolved by a third investigator (C.I.V.).

\section{Summary measures}

The primary outcome was the development of grades 1 and 2 according to the RTOG criteria for radiation dermatitis. Further measurements considered in this review were odds ratios $(\mathrm{OR})$ or risk differences for dichotomous outcomes.

\section{Synthesis of results}

The overall data combination of the included studies was performed by a descriptive synthesis. Statistical pooling of data using meta-analysis was planned whenever trials were considered combinable and relatively homogeneous in relation to design, interventions, and outcomes. Heterogeneity within studies was evaluated either by considering clinical (differences about participants, type of interventions, and results), methodological (design and risk of bias), and statistical characteristics (effect of studies).

\section{Risk of bias across studies}

The quality of evidence and grading of strength of recommendations were assessed using Grades of Recommendation, Assessment, Development, and Evaluation (GRADE) [23, 24]. The criteria for this assessment were study design, risk of bias, imprecision, inconsistency, indirectness, and magnitude of effect. The quality of evidence must be characterized as high, moderate, low, or very low [24].

\section{Results}

\section{Study selection}

In phase 1 of study selection, 1257 citations were identified across six electronic databases. After the duplicated articles were removed, 972 citations remained. The results from gray literature added 44 references. A thorough screening of the abstracts was completed, and 992 references were excluded. Hand search from the reference lists of the identified studies yielded no additional studies. Thus, 24 articles remained for a full-text screening (phase 2). This process led to the exclusion of 11 studies (Appendix 2). In total, 13 articles [25-37] were selected for data extraction and qualitative synthesis (Table 1). Figure 1 (flowchart) details the process of identification, inclusion, and exclusion of studies with reasons.

\section{Study characteristics}

Table 1 summarizes the descriptive characteristics of the studies. The studies were published in Chinese [35], English [25-34, 37], and French [36], from 1980 to 2015. All selected articles were prospective clinical trials. 


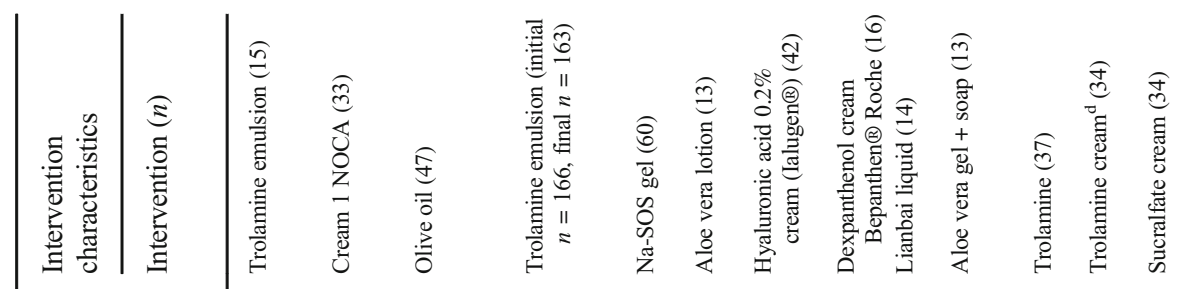

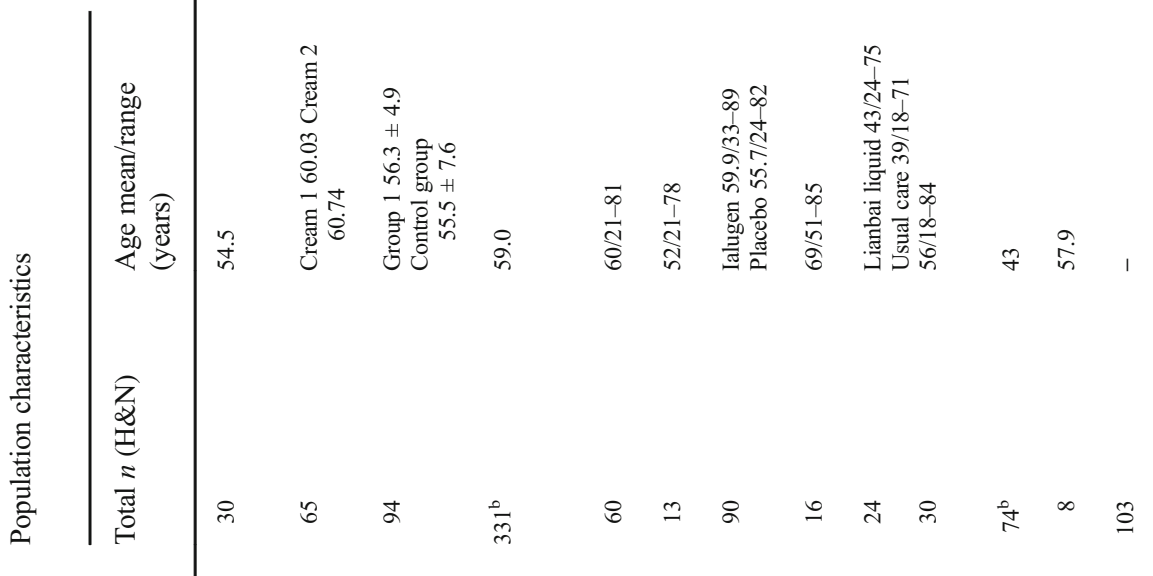
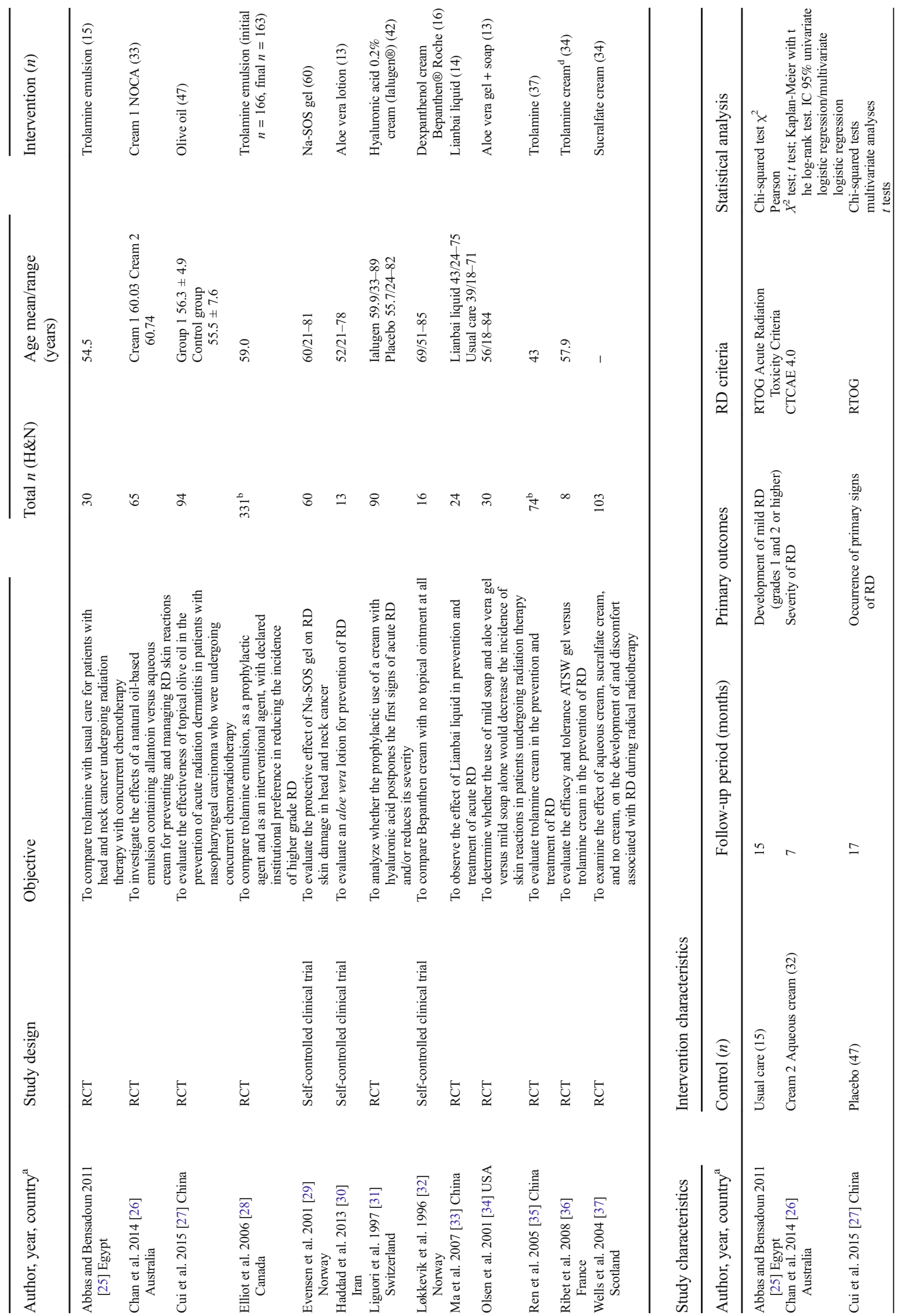


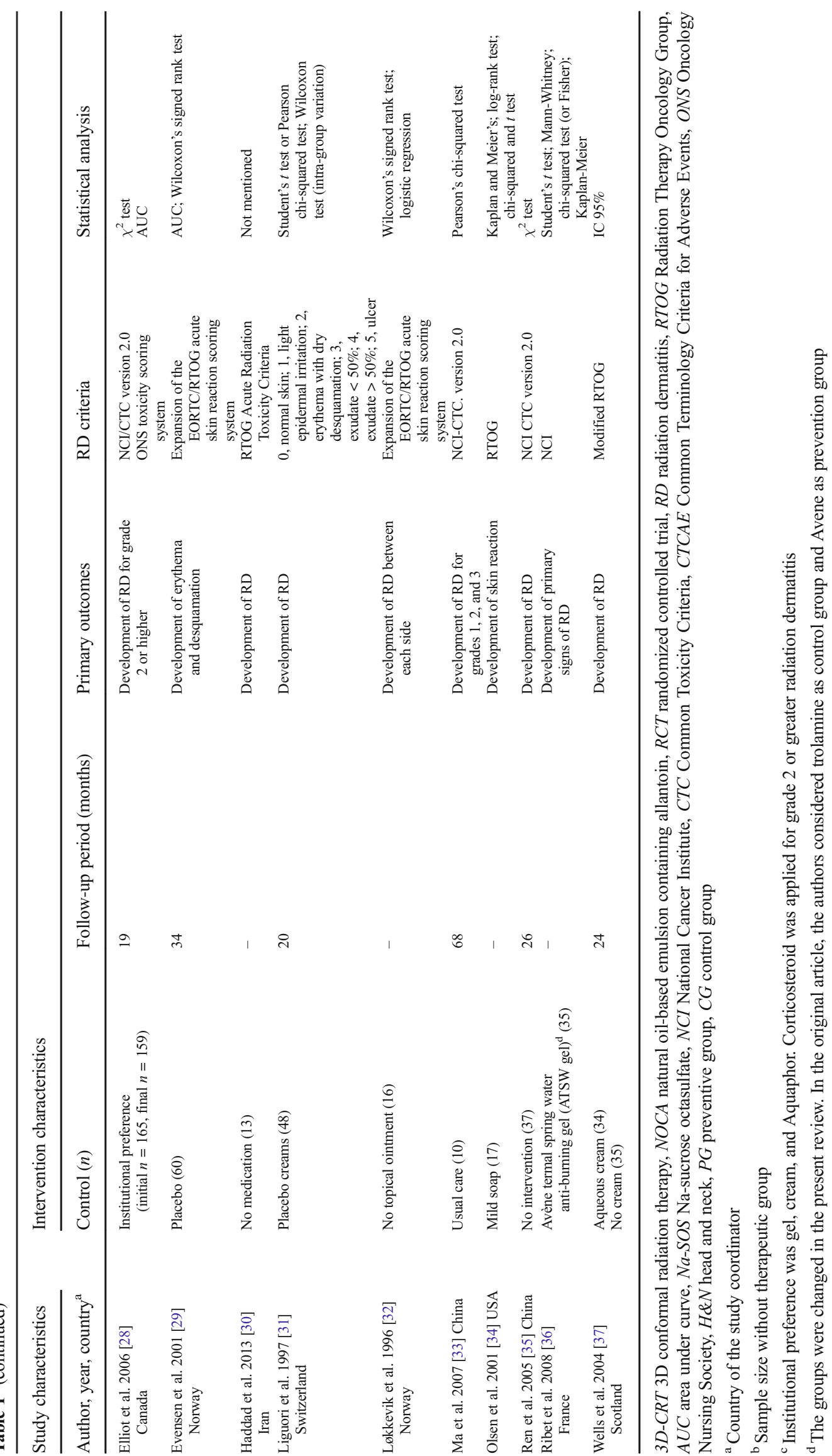


Fig. 1 Flow diagram of literature search and selection process (adapted from PRISMA)

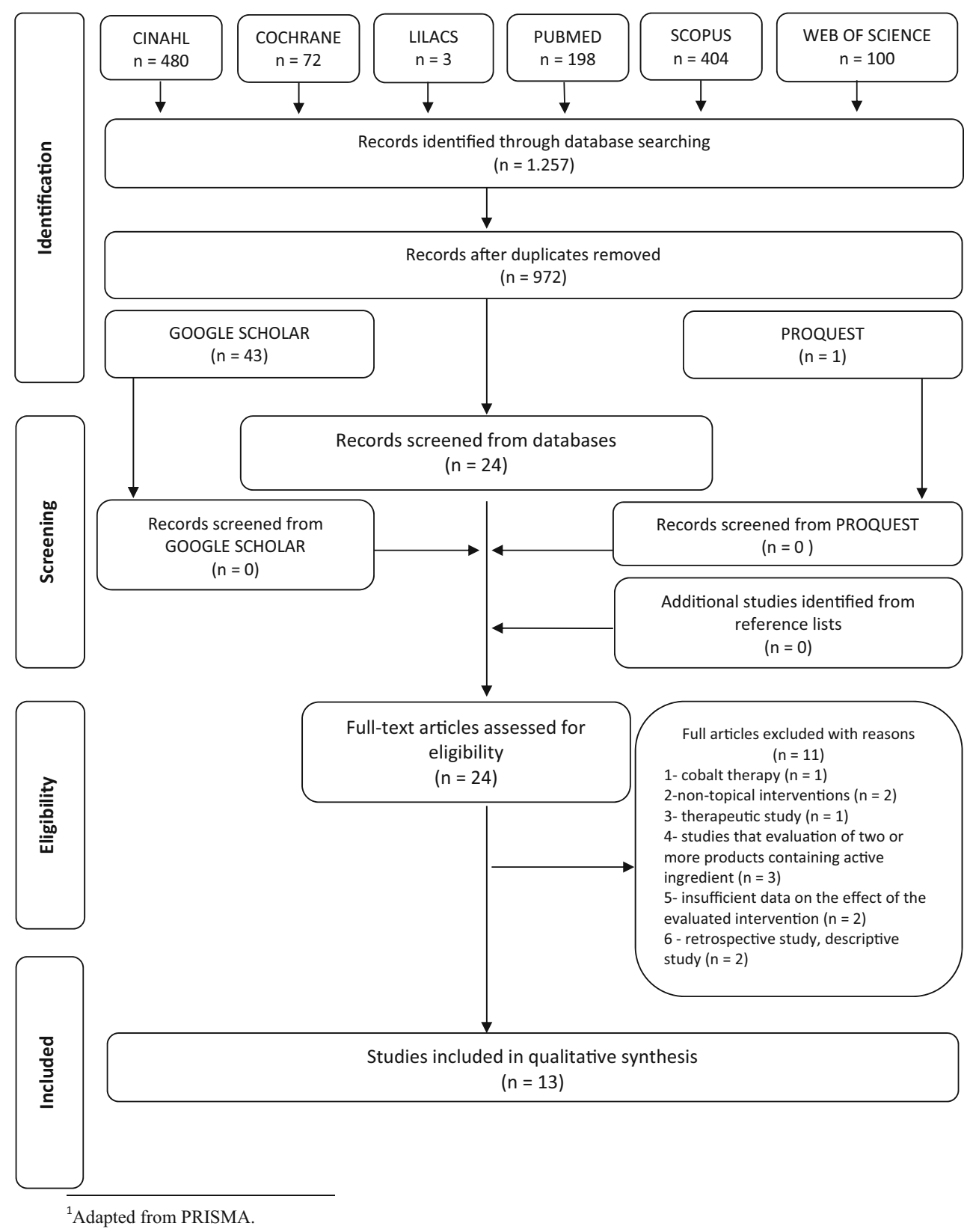

The follow-up period was mentioned in 9 out of the 13 studies (mean 25.5 months, range 7-68 months). With regard to the radiation dose received by the participants, four studies indicated minimal doses $<50 \mathrm{~Gy}$, and of these, two presented the dose averages, as follows: 59.7 Gy [29] and 54 Gy [30]. The ionizing radiation doses applied to patients are described in Fig. 2.

Seven studies included patients who also underwent concurrent chemotherapy [25-28, 30, 34, 37]. The chemotherapy protocol was specified in only two studies: cisplatin $40 \mathrm{mg} / \mathrm{m}^{2}$ weekly [25] and cisplatin $25-30 \mathrm{mg} / \mathrm{m}^{2}$ and docetaxel $25-$ $30 \mathrm{mg} / \mathrm{m}^{2}$ [27]. Exclusive RT has been reported in two studies $[32,36]$. The remaining four studies did not mention the use of chemoradiotherapy [29, 31, 33, 35].
Most studies evaluated trolamine [25, 28, 35, 36] as active principle to prevent radiation dermatitis and aloe vera [30, 34]. Other pharmacological interventions were allantoin [26], olive oil [27], Lianbai liquid [33], sucralfate [37], Na-sucrose octasulfate (Na-SOS) [29], hialuronic acid [31], and dexpanthenol [32]. Nonpharmacological topical controls were usual care/institution routine [25, 28, 33], aqueous cream [26, 37], mild soap [34], water thermal gel [35], placebo [27, $29,31]$, and no intervention [30, 32, 35].

Eight of the selected studies $(61 \%)$ included heterogeneous samples of patients with different cancer types and irradiated areas: breast, lung, pelvis, and anorectal cancer. 
Fig. 2 Ionizing radiation doses applied to patients in the included studies

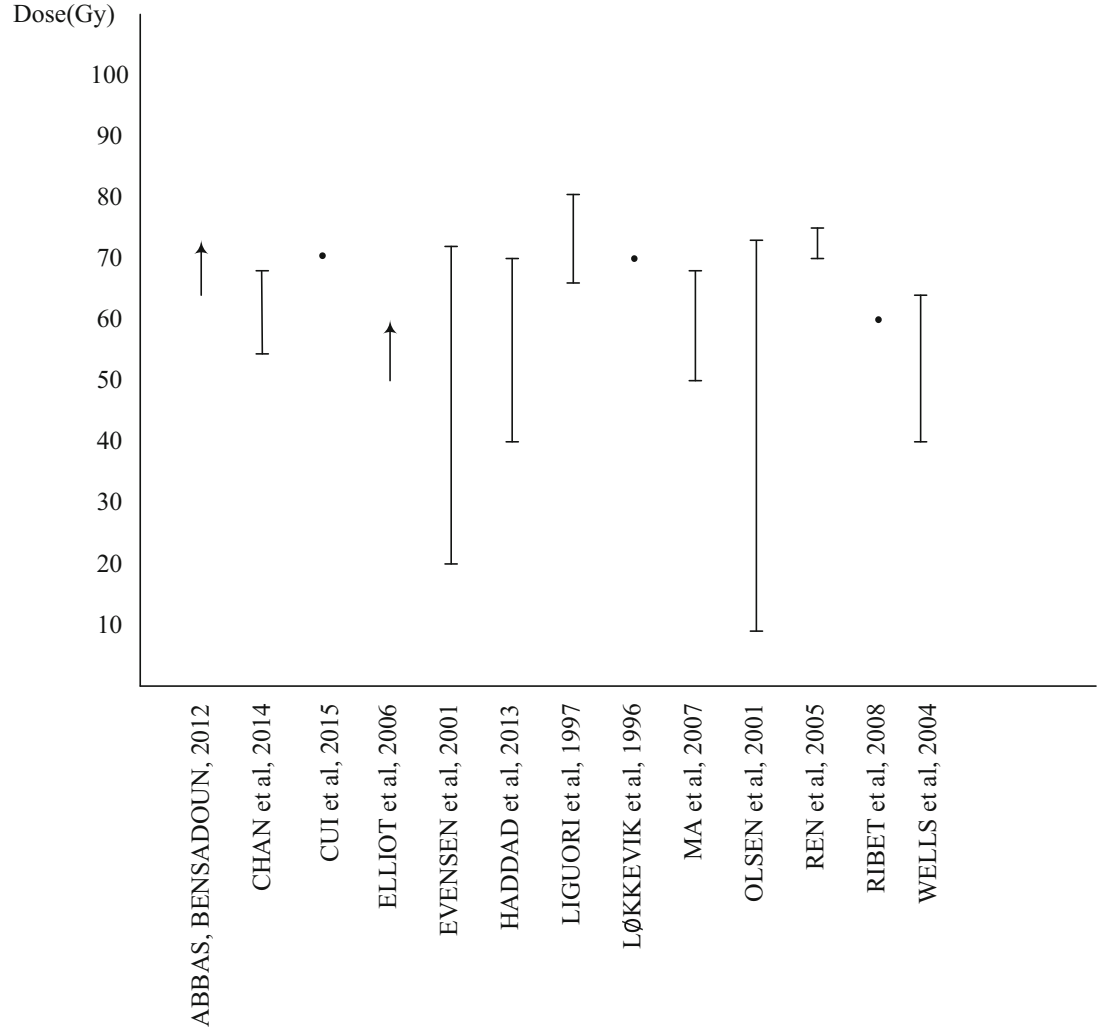

\section{Risk of bias within studies}

In this review, it was considered uncertain/unclear when those criteria were not clearly reported in the original study, with incomplete or missing information. This situation occurred in $8(61.5 \%)$ and $10(76.9 \%)$ of the included studies on the domains "random sequence generation" and "allocation concealment," respectively.

For the domain "blinding of participants and personnel," while there was an understanding that blinding of participants would not be feasible, for comparing different interventions not possible to blind and/or understanding that his absence would not alter the degree of radiation dermatitis, it was judged as low risk of bias. However, for the self-controlled studies in which the patients themselves chose the product application side or the information on the randomization was unclear [30, 32] and those in which the authors themselves concluded that lack of blinding could have caused bias [28], the risk was rated high.

The domain "incomplete outcome data" showed predominantly low risk of bias in the evaluation of the studies (10 studies; $76.9 \%$ ). This was the best result found for one single domain.

Five studies were classified as high risk of bias because they contained one or more compromised domains regarding the reliability of results $[28-30,32,36]$. Five studies were classified as uncertain risk of bias [25, 27,33-35]. Two of them received positive bias ratings, with low risk of bias in $91 \%$ of the evaluated domains $[31,37]$. Only one study presented low risk of bias in all domains evaluated [26], allowing us to ascribe the results of the study as of increased reliability. Risk of bias assessment is reported in Fig. 3.

\section{Results of individual studies}

The studies used different types of interventions to prevent radiation dermatitis and reported different results for all 13 articles. Characteristics and results of the included studies are listed in Table 1.

\section{Synthesis of results}

First of all, the 13 selected studies were analyzed by a descriptive synthesis.

Corticosteroids and non-steroidal anti-inflammatory drugs were administered to manage more severe levels of radiation dermatitis in some studies [28, 34]. Thus, given the inclusion and exclusion criteria of the current review, the graduations controlled by medications such as corticosteroids and nonsteroidal anti-inflammatory drugs were excluded from the analysis.

Regarding the rating scales, $30.7 \%$ used the RTOG scale [25, 30, 34], 30.7\% used National Cancer Institute Common Toxicity Criteria (NCI-CTC) [28, 33, 35, 36], 23\% used 


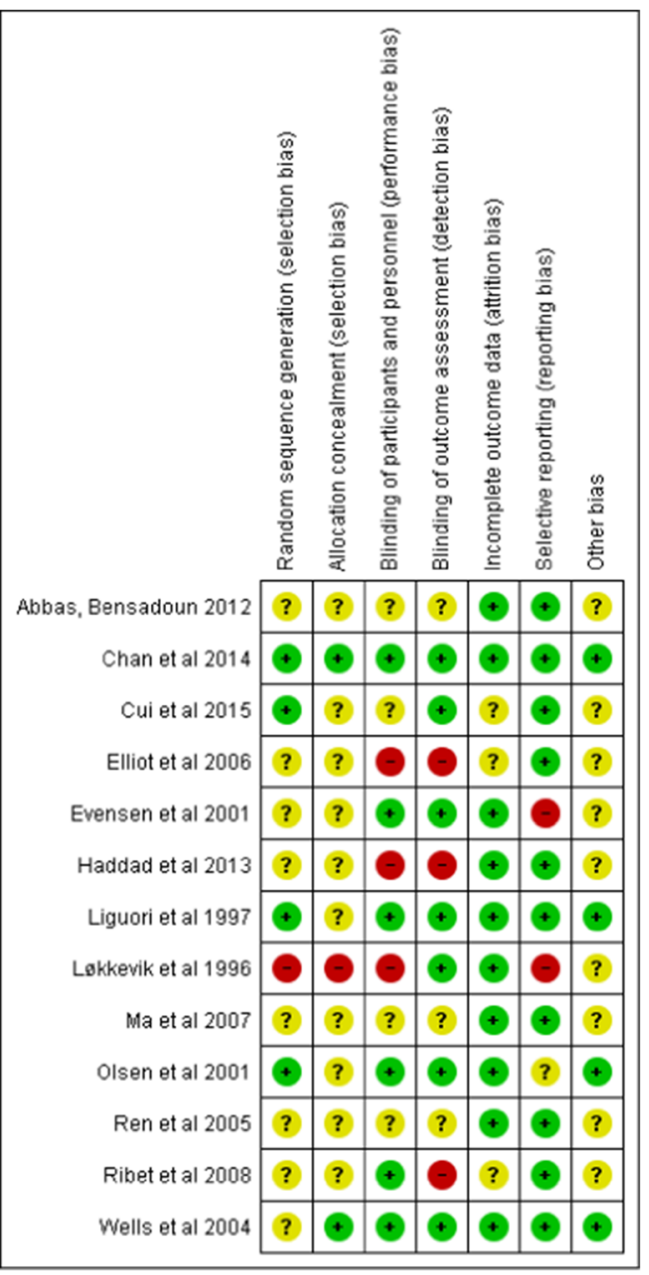

Fig. 3 Risk of bias assessment for individual studies. Question marks mean unclear; plus signs mean yes; minus signs mean no

expanded or modified scale RTOG [29, 32, 37], 7.7\% used CTCAE version 4.0 [26], and, finally, $7.7 \%$ used a scale described in the study itself [31]. One study used both NCI-CTC and ONS scales to assess the skin reactions of their patients [28].

Taking into consideration the similarity between grades 1 and 2 of both the NCI-CTC and the RTOG grading systems, the NCI-CTC grades 1 and 2 were reclassified as RTOG grades 1 and 2 for the two studies using NCI-CTC [28, 35].

In one study [28], where institutional care included hydrocortisone treatment of patients with grade 2 and above, only patients with grade 1 were included in the analysis for this review.

At the assessment of heterogeneity, some studies represented data for patients with cancers in other areas than the head and neck region [26, 29-33, 35, 36].

The studies selected for this review were considered to be relatively homogeneous, however, showed some heterogeneous points, as the interventions, controls, assessment tools of radiation dermatitis, and outcomes assessed, which influenced the quantitative analysis of data extracted from studies. Thus, there were no data that would allow a meta-analysis.

\section{Risk of bias across studies}

Overall, the quality of the evidence from the outcomes evaluated by the GRADE system was assessed as moderate (Table 2), suggesting moderate confidence in the estimated effect from the outcomes assessed. The limitations in the studies, inconsistency, and important indirect evidence were the main factors responsible for the limited quality of the evidence from studies evaluated.

\section{Discussion}

Cancer of the head and neck is relatively common. The term "head and neck cancer" comprises a large number of neoplasms from the mucosa of the upper aerodigestive tract including the oral cavity, pharynx, larynx, and sinuses [38]. Among all subtypes, carcinoma of the mouth and pharynx together rank as the sixth most common neoplasm [39]. Surgery, radiotherapy, and concurrent chemoradiotherapy have been used to manage head and neck cancer [40, 41]. This systematic review investigated evidence to evaluate the

Table 2 GRADE assessment

Quality assessment

\begin{tabular}{|c|c|c|c|c|c|c|c|}
\hline Studies $(n)$ & Type of study & Study limitations & Inconsistency & Indirectness & Imprecision & Publication bias & GRADE quality \\
\hline \multicolumn{8}{|c|}{ Outcome: acute radiation dermatitis grades 1 and 2 , according score RTOG } \\
\hline 6 & $\mathrm{RCT} /$ self-controlled & $X^{\mathrm{a}}$ & $\mathrm{X}^{\mathrm{b}}$ & $\mathrm{X}^{\mathrm{c}}$ & $\sqrt{ }$ & $\sqrt{ }$ & $\begin{array}{l}++-- \\
\text { Moderate }\end{array}$ \\
\hline
\end{tabular}

${ }^{a}$ Absence of blinding of participants and examiners in the study or uncertainty regarding its implementation and/or uncertainty about the process of randomization and blinding of the random allocation of the sample due to insufficient data

${ }^{\mathrm{b}}$ Heterogeneity

${ }^{\mathrm{c}}$ Divergence between the data presented in the study protocol and the data presented as results of the individual outcomes (e.g., absence of a specific ranking for scale previously defined) 
effects of pharmacological interventions and nonpharmacological topical controls to prevent radiation dermatitis in head and neck cancer patients.

To our knowledge, this is the first systematic review of topical interventions for the prevention of acute radiation dermatitis in patients with head and neck cancer undergoing radiotherapy. We sought to comply the criteria of AMSTAR [42] in order to increase its reliability.

The studies showed differences in baseline characteristics collected from patients and between scales used to classify radiation dermatitis. The ionizing radiation dose prescribed to patients was not described in a uniform way between studies, varying from total dose, medium dose, and dose intervals.

Delivering chemotherapy concurrently with radiation increases the severity of radiation dermatitis. However, some studies do not present this information clearly [29, 31, 33, 35], making it difficult for comparisons among subgroups who received only radiotherapy or chemoradiotherapy. Not all studies described the type of chemotherapy used, which is important as cetuximab caused more severe skin reactions than cisplatin.

This systematic review aimed to analyze studies that evaluated the effect of various topical pharmacological interventions to prevent grades 1 and 2 of radiation dermatitis according to RTOG criteria in head and neck cancer patients. There was no difference between groups of pharmacological and nonpharmacological topical controls for prevention of acute radiation dermatitis; however, vehicles or bases that do not contain the active ingredient in a formulation might have clinical relevance.

A review about topical interventions for radiation dermatitis in patients with breast cancer emphasizes the relevance of creams in reducing adverse effect and the low cost of the intervention [43]. Lotions, powders, creams, ointments, gel, and other bases are examples of vehicles for topical products. Both the vehicle and the active ingredient have action on the cutaneous response to treatment. The application time product topical is another important factor. The preferable application of the product was overnight allowing the product to remain on the skin for a longer period [21].

The usual care to prevent radiation dermatitis is also considered as a non-pharmacological care. The guidelines include hygiene orientation site, reduction of exposure and friction of the irradiated area, use of appropriate clothing, preferably cotton, avoid sun exposure and contact with extreme temperatures, as compresses, avoid itching the irradiated area, and avoid using products that have strong agents in their composition, as some types of soaps $[25,33]$. In the latter case, the option is at neutral soap [34]. Usual care and skin cleaning of irradiated area are consistent with other reviews that say that skin washing is important for the prevention of acute radiation dermatitis [9]. Product selection should also take into account the cost-effectiveness and ability to understand the patient, family, and caregivers.

To evaluate the radiation dermatitis, the criteria adopted by the main scales consist of visual measurements of signals as erythema and scaling, like the RTOG scale, modified RTOG scale, and CTCAE. A limitation of these scales is the subjectivity that can occur in radiation dermatitis classification related to own evaluator [44], leading to a significant bias when it comes to the evaluation of radiation dermatitis in multicenter studies [28].

It is important that the studies follow rigorous methodological standards. The RCTs must have to be conducted properly. The Standard Protocol Items: Recommendations for Interventional Studies (SPIRIT) [45] statements can assist in the study plan.

We recommend that in future studies, the randomization be stratified on the radiotherapy dose, so that the intervention and control groups are balanced regarding the radiotherapy fraction. There is also a need to clarify what type of chemotherapy was used concomitant to radiotherapy. Time and duration of product apply are a relevant item to describe in the methodology section of the studies. It is also important to have a definition about what is the prevention endpoint, for example, it can be the occurrence of RD (yes or not) or it can be the development of erythema, dry or moist desquamation, and edema, which are signs of $\mathrm{RD}$, generally described in some graduation scales. None of the studies included in this review took pictures to evaluate $\mathrm{RD}$, but it can be a good strategy to evaluate the progression or regression of the signs of RD.

\section{Conclusion}

There was no strong evidence indicating differences between topical pharmacological interventions and nonpharmacological topical controls related to the prevention of acute radiation dermatitis among patients with head and neck cancer undergoing radiotherapy.

Patients with head and neck cancer are usually more susceptible to develop radiation dermatitis because they are commonly exposed to high doses of radiation and combined treatment. An effort is needed in conducting studies with appropriate methodological rigor and to evaluate topical interventions with homogeneous samples.

\section{Compliance with ethical standards}

Conflict of interest The authors declare that they have no conflict of interest.

\section{References}

1. McQuestion M (2011) Evidence-based skin care management in radiation therapy: clinical update. Semin Oncol Nurs 27:e1-e17. doi:10.1016/j.soncn.2011.02.009

2. Wong RK, Bensadoun RJ, Boers-Doets CB, Bryce J, Chan A, Epstein JB, Eaby-Sandy B, Lacouture ME (2013) Clinical practice guidelines for the prevention and treatment of acute and late radiation reactions from the MASCC Skin Toxicity Study Group. 
Support Care Cancer 21:2933-2948. doi:10.1007/s00520-0131896-2

3. Häfner MF, Fetzner L, Hassel JC, Debus J, Potthoff K (2013) Prophylaxis of acute radiation dermatitis with an innovative FDAapproved two-step skin care system in a patient with head and neck cancer undergoing a platin-based radiochemotherapy: a case report and review of the literature. Dermatology 227:171-174. doi: $10.1159 / 000353974$

4. Cabezón-Gutiérrez L, Khosravi-Shahi P, Escobar-Álvarez Y (2012) Management of dermatitis in patients with locally advanced squamous cell carcinoma of the head and neck receiving cetuximab and radiotherapy. Oral Oncol 48:293-297. doi:10.1016/j. oraloncology.2011.10.019

5. Franco P, Potenza I, Moretto F, Segantin M, Grosso M, Lombardo A, Taricco D, Vallario P, Filippi AR, Rampino M, Ricardi U (2014) Hypericum perforatum and neem oil for the management of acute skin toxicity in head and neck cancer patients undergoing radiation or chemo-radiation: a single-arm prospective observational study. Radiat Oncol 9:297. doi:10.1186/s13014-014-0297-0

6. Bensadoun RJ, Humbert P, Krutman J, Luger T, Triller R, Rougier A, Seite S, Dreno B (2013) Daily baseline skin care in the prevention, treatment, and supportive care of skin toxicity in oncology patients: recommendations from a multinational expert panel. Cancer Manag Res 5:401-408. doi:10.2147/CMAR.S52256

7. Zenda S, Ishi S, Akimoto T, Arahira S, Motegi A, Tahara M, Hayashi R, Asanuma C (2015) DeCoP, a Dermatitis Control Program using a moderately absorbent surgical pad for head and neck cancer patients receiving radiotherapy: a retrospective analysis. Jpn J Clin Oncol 45:433-438. doi:10.1093/jjco/hyv010

8. O'Donovan A, Coleman M, Harris R, Herst P (2015) Prophylaxis and management of acute radiation-induced skin toxicity: a survey of practice across Europe and the USA. Eur J Cancer Care (Engl) 24:425-435. doi:10.1111/ecc.12213

9. Bolderston A, Lloyd NS, Wong RK, Holden L, Robb-Blenderman L, Supportive Care Guidelines Group of Cancer Care Ontario Program in Evidence-Based Care (2006) The prevention and management of acute skin reactions related to radiation therapy: a systematic review and practice guideline. Support Care Cancer 14: 802-817. doi:10.1007/s00520-006-0063-4

10. Chan RJ, Webster J, Chung B, Marquart L, Ahmed M, Garantziotis S (2014) Prevention and treatment of acute radiation induced skin reactions: a systematic review and meta-analysis of randomized controlled trials. BMC Cancer 14:53. doi:10.1186/1471-2407-1453

11. Koukourakis GV, Kelekis N, Kouvaris J, Beli IK, Kouloulias VE (2010) Therapeutics interventions with anti-inflammatory creams in post radiation acute skin reactions: a systematic review of most important clinical trials. Recent Patents Inflamm Allergy Drug Discov 4:149-158

12. Meghrajani CF, Co HC, Ang-Tiu CM, Roa FC (2013) Topical corticosteroid therapy for the prevention of acute radiation dermatitis. Expert Rev Clin Pharmacol 6:641-649. doi:10.1586 /17512433.2013.841079

13. Richardson J, Smith JE, McIntyre M, Thomas R, Pilkington K (2005) Aloe vera for preventing radiation-induced skin reactions: a systematic literature review. Clin Oncol (R Coll Radiol) 17:478484. doi:10.1016/j.clon.2005.04.013

14. Salvo N, Barnes E, van Draanen J, Stacey E, Mitera G, Breen D, Giotis A, Czarnota G, Pang J, De Angelis C (2010) Prophylaxis and management of acute radiation induced skin reactions: a systematic review of the literature. Curr Oncol 17:94-112

15. Zhang Y, Zhang S, Shao X (2013) Topical agent therapy for prevention and treatment of radiodermatitis: a meta-analysis. Support Care Cancer 21:1025-1032. doi:10.1007/s00520-012-1622-5

16. Moher D, Liberati A, Tetzlaff J, Altman DG, PRISMA Group (2009) The PRISMA group. Preferred reporting items for systematic reviews and meta-analyses: the PRISMA statement. Ann Intern Med 151:264-269

17. PROSPERO (2015) International prospective register of systematic reviews. http://www.crd.york.ac.uk/PROSPERO/. 2015. Accessed 30 April 2015

18. Chan RJ, Larsen E, Chan P (2012) Re-examining the evidence in radiation dermatitis management literature: an overview and a critical appraisal of systematic reviews. Int J Radiat Oncol Biol Phys 84:e357-e362. doi:10.1016/j.ijrobp.2012.05.009

19. Cox JD, Stetz J, Pajak TF (1995) Toxicity criteria of the Radiation Therapy Oncology Group (RTOG) and the European Organization for Research and Treatment of Cancer (EORTC). Int J Radiat Oncol Biol Phys 31:1341-1346

20. Common Terminology Criteria for Adverse Events (CTCAE). http://evs.nci.nih.gov/ftp1/CTCAE/CTCAE_4.03_2010-06-14_ QuickReference_8.5x11.pdf. Accessed 26 September 2016

21. Lawton S (2013) Safe and effective application of topical treatments to the skin. Nurs Stand 27:50-56

22. Higgins, JPT, Green S (editors) (2011) Cochrane Handbook for Systematic Reviews of Interventions Version 5.1.0 [updated March 2011]. The Cochrane Collaboration. www.cochranehandbook.org. Accessed 30 April 2015

23. Balshem H, Helfand M, Schünemann HJ, Oxman AD, Kunz R, Brozek J, Vist GE, Falck-Ytter Y, Meerpohl J, Norris S, Guyatt GH (2011) GRADE guidelines: 3. Rating the quality of evidence. J Clin Epidemiol 64:401-406. doi:10.1016/j.jclinepi.2010.07.015

24. Schünemann H, Brożek J, Guyatt G, Oxman A editors (2013) GRADE handbook for grading quality of evidence and strength of recommendations. Updated October 2013. The GRADE Working Group, 2013 http://gdt.guidelinedevelopment. org/central_prod/_design/client/handbook/handbook.html. 30 April 2015

25. Abbas H, Bensadoun RJ (2012) Trolamine emulsion for the prevention of radiation dermatitis in patients with squamous cell carcinoma of the head and neck. Support Care Cancer 20:185-190. doi:10.1007/s00520-011-1110-3

26. Chan RJ, Mann J, Tripcony L, Keller J, Cheuk R, Blades R, Keogh S, Poole C, Walsh C (2014) Natural oil-based emulsion containing allantoin versus aqueous cream for managing radiation-induced skin reactions in patients with cancer: a phase 3, double-blind, randomized, controlled trial. Int J Radiat Oncol Biol Phys 90:756-764. doi:10.1016/j.ijrobp.2014.06.034

27. Cui Z, Xin M, Yin H, Zhang J, Han F (2015) Topical use of olive oil preparation to prevent radiodermatitis: results of a prospective study in nasopharyngeal carcinoma patients. Int J Clin Exp Med 8:1100011006

28. Elliott EA, Wright JR, Swann RS, Nguyen-Tân F, Takita C, Bucci MK, Garden AS, Kim H, Hug EB, Ryu J, Greenberg M, Saxton JP, Ang K, Berk L, Radiation Therapy Oncology Group Trial 99-13 (2006) Phase III Trial of an emulsion containing trolamine for the prevention of radiation dermatitis in patients with advanced squamous cell carcinoma of the head and neck: results of Radiation Therapy Oncology Group Trial 99-13. J Clin Oncol 24:2092-2097

29. Evensen JF, Bjordal K, Jacobsen AB, Løkkevik E, Tausjø JE (2001) Effects of Na-sucrose octasulfate on skin and mucosa reactions during radiotherapy of head and neck cancers - a randomized prospective study. Acta Oncol 40:751-755

30. Haddad P, Amouzgar-Hashemi F, Samsami S, Chinichian S, Oghabian MA (2013) Aloe vera For prevention of radiationinduced dermatitis: a self-controlled clinical trial. Curr Oncol 20: e345-e348

31. Liguori V, Guillemin C, Pesce GF, Mirimanoff RO, Bernier J (1997) Double-blind, randomized clinical study comparing hyaluronic acid cream to placebo in patients treated with radiotherapy. Radiother Oncol 42:155-161 
32. Løkkevik E, Skovlund E, Reitan JB, Hannisdal E, Tanum G (1996) Skin treatment with bepanthen cream versus no cream during radiotherapy: a randomized controlled trial. Acta Oncol 35:10211026

33. Ma H, Zhang X, Bai M, Wang X (2007) Clinical effects of Lianbai liquid in prevention and treatment of dermal injury caused by radiotherapy. J Tradit Chin Med 27:193-196

34. Olsen DL, Raub W Jr, Bradley C, Johnson M, Macias JL, Love V, Markoe A (2001) The effect of Aloe vera gel/mild soap versus mild soap alone in preventing skin reactions in patients undergoing radiation therapy. Oncol Nurs Forum 28:543-547

35. Ren ZP, Li XM, Wu CQ, Wu D, Chen SY, Liu YJ, Zuo MS (2005) Clinical observation of trolamine cream in the prevention and treatment of acute radioactive dermatitis in patients with nasopharyngeal carcinoma. Chin J Clin Oncol 32:870-875

36. Ribet V, Salas S, Levecq JM, Bastit L, Alfonsi M, De Rauglaudre G, Talon B, Allavena C, Miot C, Boisseau JM, Faure P (2008) Interest of a sterilised anti-burning gel in radiation dermatitis: results of a comparative study. Ann Dermatol Venereol 1:5-10. doi:10.1016/S0151-9638(08)70091-7

37. Wells M, Macmillan M, Raab G, MacBride S, Bell N, MacKinnon K, MacDougall H, Samuel L, Munro A (2004) Does aqueous or sucralfate cream affect the severity of erythematous radiation skin reactions? A randomised controlled trial. Radiother Oncol 73:153162. doi:10.1016/j.radonc.2004.07.032

38. Sobin LH, Gospodarowicz MK, Wittekind C (2009) UICC TNM classification of malignant tumours, 7th edn. Wiley-Liss, New York

39. Warnakulasuriya S (2009) Global epidemiology of oral and oropharyngeal cancer. Oral Oncol 45:309-316. doi:10.1016/j. oraloncology.2008.06.002
40. Shah JP, Gil Z (2009) Current concepts in management of oral cancer surgery. Oral Oncol 45:394-401. doi:10.1016/j. oraloncology.2008.05.017

41. Salama JK, Haddad RI, Kies MS, Busse PM, Dong L, Brizel DM, Eisbruch A, Tishler RB, Trotti AM, Garden AS (2009) Clinical practice guidance for radiotherapy planning after induction chemotherapy in locoregionally advanced head and neck cancer. Int $\mathrm{J}$ Radiat Oncol Biol Phys 75:725-733. doi:10.1016/j. ijrobp.2008.11.059

42. Shea BJ, Grimshaw JM, Wells GA, Boers M, Andersson N, Hamel C, Porter AC, Tugwell P, Moher D, Bouter L (2007) Development of AMSTAR: a measurement tool to assess the methodological quality of systematic reviews. BMC Med Res Methodol 7:10. doi:10.1186/1471-2288-7-10

43. Butcher K, Willianson K (2012) Management of erythema and skin preservation; advice for patients receiving radical radiotherapy to the breast: a systematic literature review. J Radiother Pract 11:44 54. doi:10.1017/S1460396910000488

44. González-Sanchís A, Vicedo-Gonzáles A, Brualla-González L, Gordo-Partearroyo JC, Iñigo-Valdenebro R, Sánchez-Carazo J, Granero-Cabañero D, Roselló-Ferrando J, López-Torrecilla J (2014) Looking for complementary alternatives to CTCAE for skin toxicity in radiotherapy: quantitative determinations. Clin Transl Oncol 16:892-897. doi:10.1007/s12094-014-1163-0

45. Chan A-W, Tetzlaff JM, Altman DG, Laupacis A, Gøtzsche PC, Krleža-Jerić K, Hróbjartsson A, Mann H, Dickersin K, Berlin J, Doré C, Parulekar W, Summerskill W, Groves T, Schulz K, Sox H, Rockhold FW, Rennie D, Moher D (2013) SPIRIT 2013 statement: defining standard protocol items for clinical trials. Ann Intern Med 158:200-207 